\title{
A Multifunctional Composite Hydrogel as an Intrinsic and Extrinsic Coregulator for Enhanced Therapeutic Efficacy for Psoriasis
}

Jiangmei $X \mathbf{u}$

The Seventh Affiliated Hospital Sun Yat-sen University

Hao Chen

Anhui Medical University

Zhaoyou Chu

Anhui Medical University

Zhu Li

The Seventh Affiliated Hospital Sun Yat-sen University

\section{Benjin Chen}

Anhui Medical University

Jianan Sun

Anhui Medical University

Wei Lai

The Third Affiliated Hospital of Sun Yet-sun University Department of Nephrology

\section{Yan Ma}

Anhui Medical University

Yulong He

The Seventh Affiliated Hospital Sun Yat-sen University

Haisheng Qian ( $\square$ shqian@ahmu.edu.cn )

Anhui Medical University https://orcid.org/0000-0003-4903-3447

\section{Fei Wang}

The Seventh Affiliated Hospital Sun Yat-sen University

\section{Yunshen Xu}

The Seventh Affiliated Hospital Sun Yat-sen University

\section{Research Article}

Keywords: psoriasis, immunoregulation, hydrogel, methotrexate, transdermal delivery

Posted Date: January 19th, 2022

DOl: https://doi.org/10.21203/rs.3.rs-1263085/v1 
License: (c) (i) This work is licensed under a Creative Commons Attribution 4.0 International License. Read Full License

Version of Record: A version of this preprint was published at Journal of Nanobiotechnology on March 24th, 2022. See the published version at https://doi.org/10.1186/s12951-022-01368-y. 


\section{Abstract \\ Background}

Psoriasis is a chronic relapsing immunological skin disease characterized by multiple cross-talk inflammatory circuits which are relevantly associated with abnormal cross-reactivity between immune cells and keratinocytes (KCs). It may be inadequate to eradicate complicated pathogenesis only via single-mode therapy. To provide optimal combinatory therapeutics, a nanocomposite-based hydrogel was constructed by loading methotrexate (MTX) into ZnO/Ag to realize synergistic multiple target therapy of psoriasis.

\section{Results}

In this composite hydrogel, $\mathrm{ZnO}$ hybrid mesoporous microspheres were utilized both as drug carriers and ROS-scavenging nanoparticles. A proper amount of Ag nanoparticle-anchored $\mathrm{ZnO}$ nanoparticles ( $\mathrm{ZnO} / \mathrm{Ag}$ ) was functionalized with inherent immunoregulatory property. The experiments showed that $\mathrm{ZnO} / \mathrm{Ag}$ nanoparticles could exhibit a self-therapeutic effect that was attributed to reducing innate cytokine profiles by inactivating p65 in proinflammatory macrophages and abrogating secretion of adaptive cytokines in KCs by downregulating ROS-mediated STAT3-cyclin D1 signaling. A preferable antipsoriatic efficacy was achieved via topical administration of this hydrogel on the IMQ-induced psoriasis mice model, demonstrating the superior transdermal delivery and synergistic enhancement of therapeutic efficacy caused by intrinsic nanoparticles and extrinsic MTX.

\section{Conclusion}

this composite hydrogel could serve as a multifunctional, nonirritating, noninvasive and effective transcutaneous nanoagent against psoriasis.

\section{Background}

Psoriasis is a chronic autoimmunological disorder that affects approximately $2 \%$ of the global population [1-3]. Mechanistically, intricate interactions between innate and adaptive immunities, with crucial roles for proinflammatory macrophages and "activated" keratinocytes (KCs), are at the core of the pathogenesis of psoriasis [4-6]. Multiple inflammatory circuits can be mediated by these pathogenetic cells and disequilibrate the redox system of KCs, leading to inflammation and hyperproliferation of KCs $[1,7-9]$. Therefore, targeting proinflammatory macrophages and "activated" KCs simultaneously could emerge as an effective strategy for the treatment of psoriasis.

Methotrexate (MTX) is considered to be an immunosuppressive regulator involved in mediating the suppression of these pathogenetic cells. It has been systemically used in the treatment of moderate-to- 
severe psoriasis [10]. However, the inherent adverse reactions and insufficient concentration of therapeutic drug at the target lesion hamper its effect in the treatment of psoriasis [10, 11]. Fortunately, these dilemmas in conventional therapies can be circumvented by transdermal administration of MTX $[10,12,13]$. Currently, several nanocarriers, such as polymers, liposomes, metal nanoparticles and microneedles have been introduced to cater to the needs of transdermal drug delivery applications [1418]. Considering the characteristics of percutaneous treatment and better patient compliance, hydrogels have become the most competitive candidates and can be equipped with tunable functions via incorporation of various bioactive nanoparticles and MTX, as well as their good hydrophilicity and adhesiveness for psoriasis treatment [19-21]. In consideration of multiple inflammatory circuits involved in the progression of psoriasis, single-mode MTX chemotherapy may not be effective. More importantly, repeated use of high-dose MTX will cause an increasing risk of adverse reactions and drug resistance. Therefore, it is desirable to design a multifunctional composite hydrogel that could intrinsically coregulate proinflammatory macrophages and "activated" KCs, leading to synergistically enhancing therapeutic effects of extrinsic MTX.

Zinc oxide $(\mathrm{ZnO})$ has been utilized in dermatological applications for many years due to its low toxicity and low cost [22]. In our previous work, we reported a good biocompatible $\mathrm{AA}-\left[\mathrm{Zn}(\mathrm{OH})_{4}\right]^{2-}$ (denoted as $\mathrm{ZnO}$ ) hybrid mesoporous microspheres which can be served for drug delivery [23]. Herein, ZnO mesoporous microspheres were developed to incorporate MTX to achieve the sustainable release of MTX in the treatment of psoriasis. As illustrated in Scheme $1, \mathrm{ZnO} / \mathrm{Ag}(\mathrm{ZA})$ could qualify for self-therapeutic function. Ag nanoparticles were well dispersed in mesoporous microspheres, which endowed ZA with stable immunomodulatory activity to block the innate cytokine profiles by targeting macrophages. ZA could also regulate the STAT3-cyclin D1 signaling by eliminating reactive oxygen species (ROS), which restrained self-amplifying adaptive cytokine profiles. MTX-ZnO/Ag (MTX-ZA) nanoparticles were embellished with nanomicelles (NMs) and further embedded with Carbopol, denoted as Car@NMs@MTXZA hydrogel, which would show better therapeutic efficacy than MTX alone in imiquimod (IMQ)-induced psoriatic mice. In vivo and in vitro experiments, including qRT-PCR, ELISA and immunofluorescence analyses, were demonstrated. As expected, this composite hydrogel could optimize the therapeutic effects against psoriasis via synergistic multitherapy.

\section{Results And Discussion}

\section{Synthesis and charactrization of the multifunctional Car@NMs@MTX-ZA composite hydrogel}

As shown in Fig. 1a-b, the scanning electron microscope (SEM) and transmission electron microscopy (TEM) images showed that $\mathrm{ZnO} / \mathrm{Ag}$ (ZA) hybrid microspheres with ca. $200 \mathrm{~nm}$ in diameter were prepared. Scanning transmission electron microscopy (STEM) and elemental mapping images indicated that the elements of $\mathrm{Zn}, \mathrm{Ag}$ and $\mathrm{O}$ were homogeneously distributed in the overall microspheres (Fig. 1c-f). As previously described [16], the ${ }^{1} \mathrm{H}-\mathrm{NMR}$ spectra illuminated that the PCL-PEG-PCL-based nanomicelles 
(NMs) were synthesized (Fig. S1a). FT-IR spectra (Fig. 1g) showed that the MTX was successfully introduced into the prepared nanomicelles. SEM image showed the spherical morphology of NMs@MTXZA (Fig. S1b). The dynamic light scattering (DLS) and zeta potential of NMs@MTX-ZA exhibited an average size distribution of 202 nm and -19.63 mV (Fig. 1h and Fig. S2). After NMs@MTX-ZA was introduced into Carbopol, the obtained Car@NMs@MTX-ZA hydrogel showed a darker shade of yellow in appearance, and its typical pore structures were recorded by SEM (Fig. 1i). The curve of G'/G" and viscosity measurements showed this hydrogel was successfully synthesized. Additionally, energy dispersive spectroscopy (EDS) and elemental mapping indicated that ZA was homogenously distributed in the as-prepared hydrogel (Fig. 1j-k, Fig. S3 and S4).

In this hydrogel, the sustained release behavior of MTX in MTX-ZA was expected. The loading capacities of MTX in NMs@MTX-ZA and NMs@MTX were 22.95\% and 16.86\%, respectively. In vitro release studies were conducted to evaluate the release of MTX from various formulations in phosphate buffered saline at $\mathrm{pH}=7.4$. Approximately 74\% free MTX in NMs@MTX was released within $24 \mathrm{~h}$, nearly 50\% was produced from NMs@MTX-ZA, and only 40.61\% was released from the Car@NMs@MTX-ZA hydrogel (Fig.1m and Fig. S5a-b). Consistent with our anticipation, the Car@NMs@MTX-ZA hydrogel exhibited an optimal sustained release manner of MTX. This was primarily attributed to the controllable release performance of ZA [22].

\section{Self-therapeutic $\mathrm{ZnO} / \mathrm{Ag}$ nanoparticles effectively dampened the innate cytokine profiles by restraining the phosphorylation of p65 in proinflammatory macrophages}

Ag nanoparticles (Ag NPs) have immunomodulatory properties by elaborately controlling their content $[24,25]$. In this work, AA-[Zn(OH) $\left.]_{4}\right]^{2-}$ (denoted as $\mathrm{ZnO}$ ) hybrid mesoporous microspheres loaded with different amounts of Ag nanoparticles $(0.01 \%, 0.1 \%$ and $1 \mathrm{wt} \%)$ were prepared according to the protocol reported by our group [26]. Their actual amount of $\mathrm{Ag}$ was determined by inductively coupled plasma optical emission spectroscopy (ICP-OES), as shown in Table S1. The ICP-OES results of $\mathrm{Ag}^{+}$ions released from them at $24 \mathrm{~h}$ and CCK-8 showed that the ZA with $0.1 \mathrm{wt} \%$ of Ag might have better anti-inflammatory activity with an ultralow concentration of $\mathrm{Ag}^{+}$(approximately $0.20 \mathrm{ppb}$ ) and no cell cytotoxicity (Fig. $\mathbf{S 6}$ and S7) $[24,27]$.

According to the above data, we hypothesized that $0.1 \mathrm{wt} \%$ ZA could disrupt the induction of innate inflammatory cytokines, such as TNF-a, IL-23, IL-1区, and IL-6, in proinflammatory macrophages via limiting phosphorylated p65 (p-p65). To verify this hypothesis, cellular inflammatory models were employed in vitro. Nile red (NR) was used as a fluorescent substance to verify the endocytosis of THP-1 cells by confocal laser scanning microscopy (CLSM) (Fig. S8). Then, we used LPS and IFN- $\gamma$ to stimulate macrophages (denoted as M1 cells), and qRT-PCR analysis revealed that both $0.01 \mathrm{wt} \% \mathrm{ZA}$ and $0.1 \mathrm{wt} \%$ ZA significantly suppressed the mRNA expression of psoriatic cytokines, including TNF-a, IL-23, IL-1区 and IL-6 (Fig. 2a and Fig. S9). Moreover, we further addressed whether ZA could prevent IL-23-driven macrophages from secreting the unique cytokines TNF- $a$ and IL-17A [4]. Interestingly, IL-23 induced TNF-a mRNA expression in macrophages was downregulated by $0.01 \mathrm{wt} \%$ ZA and $0.1 \mathrm{wt} \%$ ZA, but IL-17A mRNA 
was only downregulated in the $0.1 \mathrm{wt} \%$ ZA-treated groups (Fig. S10). Consistent with the data in Fig. S6, $0.1 \mathrm{wt} \%$ ZA had a relatively better performance than $0.01 \mathrm{wt} \%$ ZA in suppressing the activation of proinflammatory macrophages, representing the optimal content of Ag. Therefore, $0.1 \mathrm{wt} \% \mathrm{ZA}$ was chosen to perform all experiments. The protein expression of related innate cytokines from $\mathrm{M} 1$ cells was evaluated by ELISA analysis. As shown in Fig. 2b, ZA treatment groups markedly reduced the expression of the innate cytokines TNF-a, IL-23, IL-1区, and IL- 6 in proinflammatory M1 cells. For LPS-induced RAW264.7 cells, 0.1 wt\% ZA treatment exhibited a similar trend (Fig. S11a-b). In addition, western blot analysis revealed that ZA could downregulate the phosphorylation of p65 in M1 cells (Fig. 2c-d), thus disrupting the induction of innate immunity. Moreover, innate cytokines generated from proinflammatory macrophages directly target effector KCs, leading to the production of inflammatory mediators, such as h-BD2, S100A7, CXCL1 and IL-36R [28]. These inotropic cytokines, in turn, recruit excessive macrophages infiltration to form an innate cytokine loop in psoriatic lesions $[6,29]$. To further identify the comprehensive effects of ZA on the innate cytokine loop between macrophages and $\mathrm{KC}$, we respectively collected supernatants from M1 cells with and without ZA treatment as the conditional medium (CM), labeled as control, M1, M1+ZA. HaCaT cells with CM co-culture were subjected to qRT-PCR analysis to monitor the inflammatory mediators released from HaCaT cells. Compared with the M1 condition coculture group, the expression of inflammatory mediators, h-BD2, S100A7, CXCL1, and IL-36R, were inhibited in the M1+ZA group, signifying the blockade of the autoimmune loop on KCs involved in the amplification of the innate immune response (Fig. 2e). These observations indicated that ZA, as an immunomodulator, could intrinsically block the operation of innate cytokine profiles crosstalk between macrophages and KCs.

\section{Self-therapeutic ZnO/Ag nanoparticles antagonized cyclin D1 expression and K17/cytokine autoimmune loop through eliminating ROS-induced STAT3 phosphorylation in psoriasiform keratinocytes}

Parallelly, targeting "activated" KCs is another effective strategy against psoriasis. The cytokines TNF-a and IL-17A can stimulate hyperkeratinization and adaptive cytokines secretion in KCs by disturbing the oxidant-antioxidant system [30, 31]. Signal transducer and activator of transcription 3 (STAT3), as an essential transcription factor in KCs activated by detrimental ROS [30, 32, 33], can induce the expression of K17 and cyclin D1, which is responsible for the G1/S transition in the cell cycle, leading to the excessive proliferation of $\mathrm{KCs}[34,35]$. Meanwhile, $\mathrm{K} 17$ is recognized by autoreactive $\mathrm{T}$ cells, aggravating the $\mathrm{K} 17 /$ cytokine autoimmune loop in adaptive immunity of psoriasis [36]. Therefore, it is imperative to quench the production of ROS in pathogenetic KCs. As shown in the Fig. S12, the results of the ABTS test suggested that ZA and ZnO had comparable ROS scavenging abilities [37, 38]. Therefore, we speculated that ZA could inhibit cyclin D1 expression and block the self-amplifying K17/cytokine autoimmune loop on KCs by eliminating noxious ROS-induced STAT3 phosphorylation.

Herein, a psoriasiform KCs model was employed in vitro to validate our speculation. As shown in Fig. 3ab, ZA could be effectively assimilated in $\mathrm{HaCaT}$ cells and exhibited favorable cell biocompatibility. The combination of TNF-a and IL-17 (termed as M2), was used to stimulate HaCaT cells as a psoriasiform KCs model [39]. M2-evoked intracellular ROS production was detected using a standard fluorescent probe, 
2',7'-dichlorofluorescein diacetate (DCFH-DA). Compared with HaCaT cells challenged by M2, both the ZAand $\mathrm{ZnO}$-pretreated groups exhibited low intensity green fluorescence, implying that they had comparable effects on obliterating excessive ROS in M2-stimulated KCs (Fig. 3c). On account of these results, we deeply explored the potential molecular mechanism of the ZA. As anticipated, the western blotting analysis demonstrated that the ZA treatment group remarkably decreased the phosphorylation of STAT3 compared with the M2-treated group, accompanied by the reduction of the downstream gene expression of $\mathrm{K} 17$ and cyclin D1. While the ZnO-treated group showed an inferior effect to ZA treatment group (Fig. 3d-f). In parallel, the qRT-PCR data showed that ZA treatment significantly downregulated chemokines, including CXCL1, CCL20 and IL-1区 (Fig. S13). Therefore, our hypothesis was demonstrated by the abovementioned data.

\section{The decoration of nanomicelles affected the composite hydrogel penetration in psoriatic skin}

Inspired by the results of experiments in vitro, the Polycaprolactone-Polyethyleneglycol-Polycaprolactone (PCL-PEG-PCL)-based nanomicelles were engineered to endow MTX-ZA with promoted ability in transdermal permeation, and then they were embedded into the hydrogel formulation to suit for external skin application. Before exploring the therapeutic effects of different hydrogels, we firstly examined the skin penetration and irritation of our composite hydrogels in mice model. To compare the skin permeability performance of the Car@MTX-ZA hydrogel with that of the Car@NMs@MTX-ZA hydrogel,we analyzed the biodistribution and accumulation of MTX-ZA in different cutaneous layers of the psoriatic lesion using CLSM. As shown in Fig. 4a, confocal images revealed that after $0.5 \mathrm{~h}$ of treatment, the red fluorescence of the $\mathrm{N}_{3}$ group was found under SC, but the intensity of $\mathrm{F}_{3}$ was negligible. After $2 \mathrm{~h}$ and $6 \mathrm{~h}$ of topical administration, $\mathrm{N}_{3}$ displayed a higher fluorescent signal in the dermis than $\mathrm{F}_{3}$ in a timedependent manner. After $24 \mathrm{~h}$, the deposition of MTX-ZA in $\mathrm{N}_{3}$ remained slight in the dermis but was undetectable in $F_{3}$, indicating that a particle size of $200 \mathrm{~nm}$ could permeate the epidermis [40], and the existence of nanomicelles had provided them with enhanced penetration depth and prolonged residence duration [16]. Notably, it is well known that particles with diameters of approximately few hundred nanometers can be effectively deliver into skin via skin appendages [41]. Consistency with Fig. 4a, a higher red fluorescence in hair follicles was observed. Altogether, these accumulation findings illustrated that the Car@NMs@MTX-ZA hydrogel efficiently penetrated the SC, even with a thickened epidermis in psoriatic lesions. Considering that topical MTX and metal nanoparticles might cause skin irritation and allergenic dermatitis $[42,43]$, a skin irritation study was performed to estimate the sensitization of these hydrogels. The irritation scores were the highest in the 1\% 2,4-dinitrochlorobenzene (DNCB)-induced contact dermatitis groups (Fig. $4 \mathrm{~b}$ ). The $\mathrm{F}_{1}$ group showed slight scaly symptoms, and the other groups were negligible (Fig. S14 and S15). Corresponding skin histopathological examination of these various groups was also performed, and the results were presented in Fig. 4b and Fig. S15. The DNCB group revealed that confluent nests of apoptotic keratinocytes, epidermal regenerative changes and moderate perivascular infiltrate of lymphocytes in the superficial dermis. Minor proliferation of keratinocytes and subtle infiltration of leukomonocytes in the dermis were shown in $F_{1}$ (Fig. S15), and the remaining groups 
were without histopathological changes. Collectively, these results illustrated that our composite hydrogels had satisfactory biocompatibility and were hypoallergenic for skin application.

\section{Efficacy of the composite hydrogel transdermal therapy on IMQ-induced psoriasiform skin inflammation}

The above findings suggested that the Car@NMs@MTX-ZA hydrogel with a self-therapeutic efficacy could better alleviate proinflammatory effects and relieve psoriasis symptoms. Hence, we established an IMQ-induced psoriatic mouse model to examine the effects of Car@NMs@MTX-ZA hydrogel in vivo [44]. As shown in Fig. 5a, topical administration of $5 \%$ imiquimod cream and concurrent application of different formula hydrogels were conducted with daily doses for six consecutive days, while the model group (IMQ) was managed with blank Carbopol hydrogel. Among these various treatments, $\mathrm{N}_{3}$ exhibited the most remarkable reduction of epidermal thickness, PASI scores and HE Baker grading (acanthosis and Munro microabscesses in epidermis, angiotelectasis and mononuclear cells infiltration in dermis), while the mouse weight remained constant, presenting superior efficacy to other groups (Fig. 5b-g). Likewise, the spleen length and spleen weight index, indicators of systemic immune responses, were also significantly reduced in $\mathrm{N}_{3}$ (Fig. S16a-b). A remarkable decrease in the number of $\mathrm{KI}^{2} 7^{+} \mathrm{KCs}$, a cell proliferation marker, was observed in the $\mathrm{N}_{3}$ (Fig. 5h and S16c). An equivalent amount of free MTX was also loaded on $N_{1}$, but it did not appear to be nearly as effective as $N_{3}$, which was attributed to the intrinsic anti-inflammation of ZA. In comparison with other groups, ZnO only showed minor downregulation of all the evaluation indexes. As depicted in Fig. 5b-g and S17a-h, for all groups not coated by PCL-PEG-PCL $\left(\mathrm{F}_{1}-\mathrm{F}_{3}\right)$, their therapeutic effects were not matched for the PCL-PEG-PCL decorated groups $\left(\mathrm{N}_{1}-\mathrm{N}_{3}\right)$, in terms of all the evaluation metrics. What's more, MTX and ZA alone presented almost the same therapeutic effects, ascertaining that ZA could be identified as a sort of selftherapeutic nanoparticle without side events of MTX. Taken together, this Car@NMs@MTX-ZA hydrogel was conferred with good comprehensive properties, especially optimized noninvasive transdermal drug delivery performance and augmentation of topical MTX therapeutic effects.

\section{The composite hydrogel ameliorated multiple psoriatic cytokines and the infiltration of proinflammatory macrophages in lesion skin}

To further understand the mechanisms of different hydrogels, the psoriatic cytokines in lesioned skin were measured by qRT-PCR and ELISA analysis to evaluate the ameliorative effects of the Car@NMs@MTX-ZA hydrogel. The results showed that $\mathrm{N}_{3}$ prominently suppressed the mRNA and protein expressions of TNF-a, IL-23, IL-1区 and IL-6 compared with the other groups (Fig. 6a-b). Next, we prepared to detect infiltrating proinflammatory macrophages via co-stained with F4/80 (the macrophage surface marker) and p-p65. Immunofluorescence assay showed that $\mathrm{N}_{1}$ and $\mathrm{N}_{2}$ downregulated phosphorylation of p65 in proinflammatory macrophages, further confirming the anti-inflammatory ability of ZA, but their effects were not as good as those of $\mathrm{N}_{3}$ (Fig. 6c). Thus, all these data validated that ZA possessed an immunomodulatory function, which could fortify the therapeutic effect of extrinsic MTX. Encouraged by the results of the biological activity of ZA in vitro, whether ZA could block K17 immune loop in activated 
KCs was further examined in vivo. Immunofluorescence analysis showed that the positive fluorescence intensity of K17 in $N_{3}$ was fiercely reduced compared with that in $N_{1}$ and $N_{2}$. Meanwhile, $N_{1}$ and $N_{2}$ displayed comparable downregulation. These results suggested a significant blockade effect of $\mathrm{N}_{3}$ in the IMQ-induced psoriatic skin inflammatory circuit (Fig. 6d). In addition, the predominant biocompatibility of nanomaterials in vivo is a precondition for their biomedical applications. Therefore, the toxicology of the Car@NMs@MTX-ZA hydrogel was systematically studied by collecting skin, serum and major internal organs. As data presented in Fig. S18a-d and S19a-d, after six days of treatment with these hydrogels, no apparent pathological changes were observed, regardless of the main organs, blood chemistry or hematology, indicating that these hydrogels only caused ignorable biotoxicity at the therapeutic dosage in vivo. Moreover, $\mathrm{Ag}^{+}$content was measured in skin tissue and the main organs at days 1,7 , and 28 after the final local treatment. The $\mathrm{Ag}^{+}$was mainly distributed in skin tissue, while lower $\mathrm{Ag}$ content accumulated in internal organs. At day $7, \mathrm{Ag}^{+}$was almost excreted from the skin and each organ (Fig. S20).

\section{Conclusion}

In conclusion, a multifunctional composite hydrogel with satisfactory comprehensive properties was constructed and based on $\mathrm{ZnO} / \mathrm{Ag}$ nanoparticles with inherent abilities for immunoregulation. In vitro experiments showed that $\mathrm{ZnO} / \mathrm{Ag}$ nanoparticles inhibited the activation of p65 in proinflammatory macrophages and suppressed ROS-induced STAT3-cyclin D1 signaling in KCs, resulting in simultaneous blockade of the key nodes of innate and adaptive cytokine networks. In addition, $\mathrm{ZnO} / \mathrm{Ag}$ could also serve as a nano-delivery platform to optimize the sustainable release of MTX. Meanwhile, the introduction of nanomicelles was conducive to the enhancement of transdermal delivery of nanocomposites through the thickened skin layers of psoriasis area. As a result, this hydrogel potentiated the immunomodulatory function of MTX in a psoriasis-like mouse model. Conclusively, this multifunctional hydrogel is believed to be a promising nanoplatform for the transdermal delivery of MTX, characterized by remarkable antipsoriatic efficacy, the feasibility of industrial production, and biological safety for psoriasis treatment.

\section{Materials And Methods}

\section{Materials and sample characterizations}

Tin (II) 2-ethylhexanoate (92.5-100\%), Polyethylene glycol, $\varepsilon$-Caprolactone (97\%), Lipopolysaccharide (LPS), Carbopol 934, 2,4 Dinitrochlorobenzene (DNCB), Eucalyptus oil (92.5-100\%), and phorbol 12myristate 13-acetate (PMA) were purchased from Sigma Aldrich. Triethanolamine and Methotrexate were obtained from Macklin. 5\% Imiquimod (IMQ) cream was purchased from Sichuan Mingxin Pharmaceuticals. Cell Counting-Kit 8 assay (CCK-8), Reactive Oxygen Species Assay Kit and T-AOC Assay Kit were purchased from Beyotime. Penicillin-streptomycin, trypsin, phosphate-buffered saline (PBS), DMEM and RMPI medium 1640 were purchased from Invitrogen. Fetal bovine serum (FBS) was obtained from Wisent Corporation. Cytokines IFN-y, IL-23, TNF-a and IL-17A were obtained from PeproTech. STAT3, 
p-STAT3, Cytokeratin 17 and Kallikrein 7 (KLK7) antibodies were obtained from Abcam. p65 and p-p65 antibodies were obtained from Santa Cruz. F4/80 and CyclinD1 antibodies were purchased from Cell Signaling. Primers for qRT-PCR analysis were synthesized by Sangon Biotech. The morphologies, fluorescence, and chemical constitutions of the samples were determined by conventional methods according to our previous protocol or instruments [45]. Fourier transform infrared spectroscopy (FTIR) was examined by a Thermo Nicolet iN10, ${ }^{1} \mathrm{H}$ NMR was recorded on a Bruker-400 MHz spectrometer, viscosity and storage/loss modulus (G'/G") were documented using Kinexus Rheometer (Malvern Instrument, UK).

\section{Synthesis of ZnO/Ag and NMs@MTX-ZA nanoparticles}

Firstly, AA-[Zn(OH) $\left.{ }_{4}\right]^{2-}$ (denoted as $\mathrm{ZnO}$ ) were synthesized according to our previous research with some modification [23]. Briefly, $0.376 \mathrm{mmol}$ of AA and $1.96 \mathrm{mmol}$ of CTAB were dissolved using $150 \mathrm{~mL}$ deionized water and added into a $250 \mathrm{~mL}$ round-bottom flask. Next, $0.398 \mathrm{mmol}$ of HMTA and 0.398 mmol of $\mathrm{Zn}\left(\mathrm{NO}_{3}\right)^{2} \cdot 6 \mathrm{H}_{2} \mathrm{O}$ were added into the mixed solution with stirred for $30 \mathrm{~min}$, then the mixed solution was heated at $85^{\circ} \mathrm{C}$ for $10 \mathrm{~h}$ to obtain the suspension, which was washed several times using absolute ethanol and deionized water, and then dried. Subsequently, three types of $\mathrm{ZnO} / \mathrm{Ag}$ (ZA) nanoparticles $(0.01 \%, 0.1 \%$ and $1 \mathrm{wt} \% \mathrm{ZA})$ containing different $\mathrm{Ag}$ amount were prepared via ZnO hollow microspheres laden with $\mathrm{Ag}$ nanoparticles by adjusting the concentration of $\mathrm{AgNO}_{3}$ according to our previous study [26]. Nanomicelles (PCL-PEG-PCL) and PCL-PEG-PCL decorated MTX-ZA (termed as NMs@MTX-ZA) was synthesized by previously reported method with some modification [16]. Briefly, 3.74 $\mathrm{mL}$ of PCL, $4.05 \mathrm{~g}$ stannous octoate and $2 \mathrm{~g}$ of PEG were heated and stirred at $140^{\circ} \mathrm{C}$ for 24 hours. After 24 $\mathrm{h}$, the obtained copolymer was precipitated in icy distilled water, washed three times and lyophilized. Then, $50 \mathrm{mg}$ of the obtained PCL-PEG-PCL, $10 \mathrm{mg}$ of MTX and $10 \mathrm{mg}$ of ZA were dissolved in $0.5 \mathrm{~mL}$ DMSO with stirred for $6 \mathrm{~h}$. The mixed organic phase was added into $10 \mathrm{~mL}$ deionized water with continuous ultrasound for 30min, then the obtained NMs@MTX-ZA was centrifugated at 10,000 rcf for 25 minutes. The a centrifugated deposit was resuspended in deionized water and lyophilized. Likewise, NMs@MTX and NMs@ZA were synthesized using the same protocol with the MTX or ZA alone.

\section{Evaluation of antioxidant activity}

Total antioxidant capacity of $0.01 \mathrm{wt} \% \mathrm{ZA}, 0.1 \mathrm{wt} \% \mathrm{ZA}, 1 \mathrm{wt} \% \mathrm{ZA}$ and $\mathrm{ZnO}$ were tested by ABTS rapid method using the T-AOC Assay Kit referring to the instructions.

\section{Preparation of Car@NMs@MTX-ZA hydrogel}

Car@NMs@MTX-ZA, Car@NMs@MTX and Car@NMs@ZA hydrogels were obtained by loading NMs@MTX-ZA, NMs@MTX and NMs@ZA into hydrogels according to a previous report, repectivley [16]. Eucalyptus oil used as penetration enhancer was introduced into all hydrogels. Car@MTX, Car@ZA and Car@MTX-ZA were prepared in the same manner without the decoration of NMs. 


\section{In vitro MTX release from Car@NMs@MTX-ZA hydrogel}

MTX release profiles were performed in $20 \mathrm{~mL}$ phosphate buffer $\mathrm{pH} 7.4$ at $37^{\circ} \mathrm{C}$ with shaking at $120 \mathrm{rpm}$, respectively. $1 \mathrm{~mL}$ of samples loaded into dialysis membrane (3500 Da), respectively. The dialysis membrane was put into $20 \mathrm{~mL}$ of release media. At preset time intervals $(2,4,8,12,24,48,96,144$ hours), $2 \mathrm{~mL}$ of release solution was collected, and substituted with an equal amount of fresh medium to maintain unchanged total volume. The collected release mediums were subjected to UV-vis spectrophotometer to analyze MTX concentration, the $\lambda_{\max }$ of MTX was $302 \mathrm{~nm}$.

\section{Cell lines}

HaCaT, Murine macrophage RAW264.7 and human monocytic leukemia THP-1 cells were purchased from the China Center for Type Culture Collection. HaCaT and RAW264.7 cells were grown in DMEM medium; THP-1 cells were cultivated in RPMI 1640 medium, All the culture medium complemented with $10 \%$ FBS, $1 \%$ penicillin and streptomycin. All cells were not contaminated with mycoplasma. Induction of THP-1 derived inflammatory macrophages: THP-1 cells were cultured with $100 \mathrm{ng} / \mathrm{mL}$ PMA in 6-wells cell culture plates for $24 \mathrm{~h}$ to obtain differentiated and plastic-adherent M0 macrophages, the culture medium of M0 cells was replaced with fresh RPMI 1640 media to obtain resting macrophages and then challenged by $100 \mathrm{ng} / \mathrm{mL}$ LPS plus $20 \mathrm{ng} / \mathrm{mL}$ IFN- $\gamma$ to generate inflammatory macrophages (M1 cells). Establishment of in vitro psoriatic model: HaCaT cells were co-stimulated with $10 \mathrm{ng} / \mathrm{mL}$ TNF-a and 200ng/mL IL17A (denoted as M2) in DMEM for $24 \mathrm{~h}$ to recapitulate features of psoriasis [39]. The control cells used in all measurements were exposed with the culture medium containing phosphate buffered saline in similar amount as other treatments.

\section{In vitro cellular uptake and cell viability assays}

THP-1cells and HaCaT cells were incubated with Nile Red (NR)-labeled ZA NPs for $4 \mathrm{~h}$, followed by the red fluorescence images were assessed by CLSM (LMS-800, Carl Zeiss) to confirm cellular uptake of ZA NPs. THP-1 cells, HaCaT cells and RAW264.7 cells were exposed with $0.01 \mathrm{wt} \% \mathrm{ZA}, 0.1 \%$ wt ZA and $1 \%$ wt ZA for $24 \mathrm{~h}$, followed by evaluating cell viability using CCK-8 according to the kit introductions.

\section{In vitro ROS assay}

Intracellular ROS generation was estimated by DCFH-DA. Briefly, HaCaT cells $\left(2-3 \times 10^{5} \mathrm{cells} / \mathrm{mL}\right)$ were cultured into $1 \mathrm{~mL}$ culture medium of confocal plates. On the second day, the cells were pretreated with fresh DMEM medium containing $0.2 \mu \mathrm{g} / \mathrm{mL} 0.1 \mathrm{wt} \% \mathrm{ZA}$ and $0.2 \mu \mathrm{g} / \mathrm{mL} \mathrm{ZnO}$ for $18 \mathrm{~h}$, respectively. then the cells were stimulated by another $6 \mathrm{~h}$ under TNF- $\alpha$ and IL-17A stimulation. Finally, the cells were cultured with DCFH-DA for $30 \mathrm{~min}$ at $37^{\circ} \mathrm{C}$ in the dark to determine ROS level. After $30 \mathrm{~min}$, the cells were washed using phosphate buffered saline. Oxidative stress of HaCaT cells was captured by using CLSM.

\section{qRT-PCR analysis}


Total RNA from cells and mice tissues were withdrawned with TRIzol (Life technogies, 15596026) according to the kits' instructions. Genomic DNA was eliminated and cDNA was then reverse transcribed with the total RNA $(1 \mu \mathrm{g})$, a primeScript RT reagent kit (TaKaRa, RR047A) was applied to reverse transcription to synthesize cDNA. The obtained $c D N A(1 \mu \mathrm{L})$ was received to qRT-PCR analysis using Novostart SYBR qPCR SuperMix Plus (Novoprotein, E096-01B). The results were normalized to $A C T B$, and the $2^{-\triangle \triangle C t}$ method was performed to quantify. All primers were obtained from Sangon Biotech Co., Ltd.

\section{Western blot}

Samples obtained from cells were lysed, separated by electrophoresis and transferred to polyvinylidene fluoride (PVDF) membranes (MerckMinipore, IPVH00010). The proteins were incubated with the primary antibodies: GAPDH (ZSGB-BIO, TA-08, 1:10000 dilution), p-STAT3 (Abcam, Ab267373,1:2000 dilution), STAT3 (Santa Cruz, sc-8019, 1:1000 dilution), p65 (Abcam, bs-0466R, 1:1000 dilution), p-p65 (Santa Cruz, sc-136548, 1:1000 dilution), Cytokeratin 17 (Abcam, Ab51056, 1:10000 dilution), CyclinD1 (Cell Signaling Technology, 55506T, 1:1000 dilution). Goat anti-rabbit or goat anti-mouse antibodies (ZSGB-BIO, ZA-2305 or ZA-2301, 1:10000 dilution) was used to label the primary antibodies and further probed using ECL reagents (Thermo). The band intensities of the images were quantified using ImageJ.

\section{ELISA analysis}

Supernatants of cell culture and tissue homogenate were collected. The TNF-a, IL-23, IL- 6 and IL-1 $\beta$ levels were measured using the TNF-a Detection Kit (JYM0110Hu, GR2021-03), IL-23 ELISA kit (JYM0083Hu, 20210810), IL-6 ELISA kit (JYM0140Hu, GR2021-03), IL-1区 ELISA kit (JYM0083Hu, GR2021-03) according to the manufacturer's protocol.

\section{In vivo skin irritation}

Skin irritation assay was performed according to previous research with some modifications[46]. Briefly, Mice were randomized into 8 groups (3 mice in each group): control group with acetone, irritant dermatitis group with 1\% 2,4-dinitrochlorobenzene (DNCB) in acetone, other groups were treated daily $(0.5 \mathrm{~mL})$ with Car@NMs@MTX-ZA hydrogel, Car@NMs@ZA hydrogel, Car@NMs@MTX hydrogel, Car@MTX-ZA hydrogel, Car@ZA hydrogel, and Car@MTX hydrogel on the back of mice for six days, respectively. The skin potential irritation of these hydrogels was compared with the irritant dermatitis group. The control group did not manage with any treatment. Signs of skin irritation of mice were monitored every day before and after hydrogels treatment throughout the period. An overall irritation score ranging from 0 to 4 : $0=$ no difference, 1 = mild erythema, 2 = well defined erythema, 3 = strong erythema, 4 = very strong erythema. Histopathological analyses of the dorsal skin of mice were further employed to confirm the skin irritation profiles.

\section{In vivo skin penetration of Car@NMs@MTX-ZA hydrogel in psoriasis}


To evaluate the biodistribution and skin permeability of NMs@MTX-ZA in IMQ mice. On day 4, Nile red (NR)-labeled Car@NMs@MTX-ZA and Car@MTX-ZA hydrogels were applied to the dorsal skin of an IMQinduced psoriatic mouse model $\left(0.5 \mathrm{~mL} / 2 \times 3 \mathrm{~cm}^{2}\right)$. The psoriatic skin tissues were collected and then embedded into 0.C.T. compound at a time intervals of $0.5,2,6$ and $24 \mathrm{~h}$. Next, these tissues were frozen using liquid nitrogen and sliced at $7 \mu \mathrm{m}$ by Cryotome, and then were fixed with $4 \%$ paraformaldehyde. Skin sections were stained with KLK7 to distinguish from epidermis and dermis, and nuclei were counterstained with DAPI. Finally, slides were mounted by antifade mountant. The distribution of MTX-ZA in psoriatic lesions was captured by CLSM.

\section{Animals and psoriasiform model}

Six to eight-week-old female C57BL/6J mice were purchased from Ji'Nan Pengyue Laboratory Animal Breeding Co., Ltd. All mice were fed and housed in specific pathogen-free conditions of the Anhui Medical University laboratory animal center. The Ethical Committee of Anhui Medical University approved all animal experiments. (Approved number: LLSC20210077). Psoriasiform skin inflammation was established by $5 \%$ Imiquimod cream, according to the literature [44]. Female mice with shaved back hair were randomly divided into 9 groups $(n=4)$. IMQ cream $(62.5 \mathrm{mg})$ was applied daily to the shaved back area of each group except for the sham group. After $4 \mathrm{~h}$, the experimental groups were treated with various hydrogels. All treatment groups were conducted for 6 consecutive days, and mice were weighed and monitored daily for PASI (Psoriasis Area and Severity Index was employed to evaluate IMQinduced erythema, scales, and thickness). On day 7, mice were sacrificed following injection with euthanasia solution followed by skin tissue and spleen dissociation. Samples from these mice were collected immediately, flash frozen, stored at $-80^{\circ} \mathrm{C}$ for qRT-PCR and ELISA analysis, and placed into formalin for histological analysis.

\section{Histopathology, immunofluorescence and immunohistochemistry assays}

Mouse dorsal skin tissues were collected and fixed with $4 \%$ formaldehyde for $24 \mathrm{~h}$ followed by dehydrated and embedded into paraffin. Next these tissues were sectioned at $7 \mu \mathrm{m}$ and stained with H\&E. Histopathology Slices were scanned using 3DHISTECH. For immunofluorescence and immunohistochemistry, $4 \%$ formaldehyde-fixed paraffin-embedded dorsal skin sections were deparaffinized, and stained with KI67, F4/80, p-p65, and K17. The images were recorded on an Olympus microscope (IX73).

\section{In vivo toxicity estimation}

To evaluate the accumulation of $\mathrm{Ag}^{+}$in the skin and main internal organs, healthy C57BL/6J mice were topically administrated with Car@NMs@MTX-ZA hydrogels daily as described above. They sacrificed at predetermined time points (the 1st,7th, and 28th day) post treatment. The skin tissue and the main organs were collected to measure the $\mathrm{Ag}^{+}$content using ICP-OES. Another three healthy C57BL/6J mice were used for the control. blood samples $(\sim 1.0 \mathrm{~mL})$ were collected for blood biochemistry test by using the standard kits according to the manufacturer's instructions. For histological assessment, every 
mouse was sacrificed and the main organs (heart, liver, spleen, lung, and kidney) were collected for hematoxylin and eosin staining.

\section{Statistical analysis}

Statistical analysis was executed using a one-way ANOVA. The differences were considered to be statistically significant for a $p$-value $\left({ }^{\star} p<0.05,{ }^{* \star} p<0.01,{ }^{* \star *} p<0.001,{ }^{* \star *} p<0.0001\right)$.

\section{Abbreviations}

TNF-a

Tumor necrosis factor alpha-like

IL-23

Interleukin 23

IL-6

Interleukin 6

IL-36R

Interleukin 1 receptor like 2

h-BD2

Defensin beta $4 \mathrm{~A}$

S100A7

S100 calcium binding protein A7

CXCL1

CXC chemokine ligand 1

IL-1】

Interleukin 1 beta

CCL20

CC chemokine ligand 20

K17

Keratin 17.

\section{Declarations}

\section{Supplementary Information}

The experimental section, SEM images, ${ }^{1} \mathrm{H}-\mathrm{NMR}$ spectra, UV-Vis-NIR absorption spectra, skin irritation assays, serum biochemistry analysis and biodistribution of $\mathrm{Ag}^{+}$results after topical administration, and H\&E staining results for major organs are provided in the supplementary information. The Supplementary Information is available free of charge on the Journal of Nanobiotechnology Publications website at http://jnanobiotechnology.biomedcentral.com.

\section{Authors' contributions}


Jiangmei Xu and Hao Chen contributed to this work equally for designing the experiments, performing the main experimental work, analyzing the main data, and writing the manuscript. Zhaoyou Chu, Zhu Li, Benjin Chen and Jianan Sun performed part of the experimental work and analyzed the results. Wei Lai, Yan Ma, and Yulong He conceptualized the study. Haisheng Qian, Fei Wang, and Yunshen Xu conducted and revised the study and manuscript.

\section{Ethics approval and consent to participate}

The Ethical Committee of Anhui Medical University approved all animal experiments. (Approved number: LLSC20210077)

\section{Consent for publication}

All the co-authors were aware of this submission and approve for publication.

\section{Availability of data and materials}

The datasets used and analyzed during the current study are available from the corresponding author on reasonable request.

\section{Competing interests}

The authors declare no competing financial interest.

\section{Funding}

This work was financially supported by grants from the National Natural Science Foundation of China (No. 81771531, 82072862, 52172276, U20A20379), research fund from Anhui Provincial Institute of Translational Medicine (2021zhyx-B15) and Grants for Scientific Research of BSKY (No: XJ201933) from Anhui Medical University.

\section{Acknowledgements}

We greatly appreciate the support of The Seventh Affiliated Hospital, Sun Yat-sen University; the School of Biomedical Engineering, Anhui Medical University and Engineering Center of Biomedical Materials, Anhui Provincial Institute of Translational Medicine.

\section{Authors' information}

${ }^{\dagger}$ Department of Dermatovenerology, The Seventh Affiliated Hospital, Sun Yat-sen University, Shenzhen, Guangdong, P. R. China

${ }^{\ddagger}$ Department of Dermatovenerology, The Third Affiliated Hospital, Sun Yat-sen University, Guangzhou, Guangdong, P. R. China 
§School of Basic Medical Sciences, School of Biomedical Engineering, Research and Engineering Center of Biomedical Materials, Anhui Provincial Institute of Translational Medicine

${ }^{\nabla}$ Center for Digestive Disease, The Seventh Affiliated Hospital, Sun Yat-sen University, Shenzhen, Guangdong, P. R. China

\# These authors contributed equally to this work.

*Address correspondence to: shqian@ahmu.edu.cn (H. Qian), fay_wong1116@hotmail.com (F. Wang) and xuysh9@mail.sysu.edu.cn (Y. Xu)

\section{References}

1. Ghoreschi K, Balato A, Enerbäck C, Sabat R. Therapeutics targeting the IL-23 and IL-17 pathway in psoriasis. Lancet. 2021; 397(10275):754-766.

2. Armstrong AW, Read C. Pathophysiology, Clinical Presentation, and Treatment of Psoriasis: A Review. JAMA. 2020; 323(19):1945-1960.

3. Parisi R, Iskandar IYK, Kontopantelis E, Augustin M, Griffiths CEM, Ashcroft DM, Global Psoriasis A. National, regional, and worldwide epidemiology of psoriasis: systematic analysis and modelling study. BMJ. 2020; 369:m1590.

4. Hou Y, Zhu L, Tian H, Sun HX, Wang R, Zhang L, Zhao Y. IL-23-induced macrophage polarization and its pathological roles in mice with imiquimod-induced psoriasis. Protein Cell. 2018; 9(12):1027-1038.

5. Wang H, Peters T, Kess D, Sindrilaru A, Oreshkova T, Van Rooijen N, Stratis A, Renkl AC, Sunderkotter $\mathrm{C}$, Wlaschek $\mathrm{M}$ et al. Activated macrophages are essential in a murine model for $\mathrm{T}$ cellmediated chronic psoriasiform skin inflammation. J Clin Invest. 2006; 116(8):2105-2114.

6. Lowes MA, Russell CB, Martin DA, Towne JE, Krueger JG. The IL-23/T17 pathogenic axis in psoriasis is amplified by keratinocyte responses. Trends Immunol. 2013; 34(4):174-181.

7. Jiang Y, Tsoi LC, Billi AC, Ward NL, Harms PW, Zeng C, Maverakis E, Kahlenberg JM, Gudjonsson JE. Cytokinocytes: the diverse contribution of keratinocytes to immune responses in skin. $\mathrm{JCl}$ Insight. 2020; 5(20).

8. Zaba LC, Cardinale I, Gilleaudeau P, Sullivan-Whalen M, Suárez-Fariñas M, Suárez Fariñas M, Fuentes-Duculan J, Novitskaya I, Khatcherian A, Bluth MJ et al. Amelioration of epidermal hyperplasia by TNF inhibition is associated with reduced Th17 responses. J Exp Med. 2007; 204(13):3183-3194.

9. Greb JE, Goldminz AM, Elder JT, Lebwohl MG, Gladman DD, Wu JJ, Mehta NN, Finlay AY, Gottlieb AB. Psoriasis. Nat Rev Dis Primers. 2016; 2:16082. 
10. Chan ESL, Cronstein BN. Methotrexate-how does it really work? Nat Rev Rheumatol. 2010; 6(3):175-178.

11. Ye Y, Yu J, Wen D, Kahkoska AR, Gu Z. Polymeric microneedles for transdermal protein delivery. Adv Drug Deliv Rev. 2018; 127:106-118.

12. Prausnitz MR, Langer R. Transdermal drug delivery. Nat Biotechnol. 2008; 26(11):1261-1268.

13. Anselmo AC, Gokarn Y, Mitragotri S. Non-invasive delivery strategies for biologics. Nat Rev Drug Discov. 2018; 18(1):19-40.

14. Zhang Y, Yu J, Kahkoska AR, Wang J, Buse JB, Gu Z. Advances in transdermal insulin delivery. Adv Drug Deliv Rev. 2019; 139:51-70.

15. Mo R, Jiang T, Di J, Tai W, Gu Z. Emerging micro- and nanotechnology based synthetic approaches for insulin delivery. Chem Soc Rev. 2014; 43(10):3595-3629.

16. Qindeel M, Khan D, Ahmed N, Khan S, Asim Ur R. Surfactant-Free, Self-Assembled NanomicellesBased Transdermal Hydrogel for Safe and Targeted Delivery of Methotrexate against Rheumatoid Arthritis. ACS Nano. 2020; 14(4):4662-4681.

17. Kim JY, Ahn J, Kim J, Choi M, Jeon H, Choe K, Lee DY, Kim P, Jon S. Nanoparticle-Assisted Transcutaneous Delivery of a Signal Transducer and Activator of Transcription 3-Inhibiting Peptide Ameliorates Psoriasis-like Skin Inflammation. ACS Nano. 2018; 12(7):6904-6916.

18. Donnelly RF, Singh TRR, Garland MJ, Migalska K, Majithiya R, McCrudden CM, Kole PL, Mahmood TMT, McCarthy HO, Woolfson AD. Hydrogel-Forming Microneedle Arrays for Enhanced Transdermal Drug Delivery. Adv Funct Mater. 2012; 22(23):4879-4890.

19. Liang Y, He J, Guo B. Functional Hydrogels as Wound Dressing to Enhance Wound Healing. ACS Nano. 2021. doi: 10.1021/acsnano.1c04206.

20. Yan X, Fang WW, Xue J, Sun TC, Dong L, Zha Z, Qian H, Song YH, Zhang M, Gong X et al. Thermoresponsive in Situ Forming Hydrogel with Sol-Gel Irreversibility for Effective Methicillin-Resistant Staphylococcus aureus Infected Wound Healing. ACS Nano. 2019; 13(9):10074-10084.

21. Gan D, Xing W, Jiang L, Fang J, Zhao C, Ren F, Fang L, Wang K, Lu X. Plant-inspired adhesive and tough hydrogel based on Ag-Lignin nanoparticles-triggered dynamic redox catechol chemistry. Nat Commun. 2019; 10(1):1487.

22. Xiong H-M. ZnO nanoparticles applied to bioimaging and drug delivery. Adv Mater. 2013; 25(37):5329-5335. 
23. Zhang C, Liu H, Wang W, Qian H, Cheng S, Wang Y, Zha Z, Zhong Y, Hu Y. Scalable fabrication of ZnxCd1-xS double-shell hollow nanospheres for highly efficient hydrogen production. Appl Catal B. 2018; 239:309-316.

24. Yang Y, Guo L, Wang Z, Liu P, Liu X, Ding J, Zhou W. Targeted silver nanoparticles for rheumatoid arthritis therapy via macrophage apoptosis and Re-polarization. Biomaterials. 2021; 264:120390.

25. Ninan N, Goswami N, Vasilev K. The Impact of Engineered Silver Nanomaterials on the Immune System. Nanomaterials (Basel). 2020; 10(5).

26. Chen B, Jiang Y, Zhao M, Wang W, Chu Z, Huo R, Hu F, Zhou W, He T, Qian H. Ag nanoparticles decorated hybrid microspheres for superior antibacterial properties. Mater Lett. 2020; 262.

27. Chen Y, Guan M, Ren R, Gao C, Cheng H, Li Y, Gao B, Wei Y, Fu J, Sun J et al. Improved Immunoregulation of Ultra-Low-Dose Silver Nanoparticle-Loaded TiO2 Nanotubes via M2 Macrophage Polarization by Regulating GLUT1 and Autophagy. Int J Nanomedicine. 2020; 15:2011-2026.

28. Feng L, Song $P, X u F, X u L$, Shao F, Guo M, Huang W, Kong L, Wu X, Xu Q. cis-Khellactone Inhibited the Proinflammatory Macrophages via Promoting Autophagy to Ameliorate Imiquimod-Induced Psoriasis. J Invest Dermatol. 2019; 139(9):1946-1956 e1943.

29. Dainichi T, Kitoh A, Otsuka A, Nakajima S, Nomura T, Kaplan DH, Kabashima K. The epithelial immune microenvironment (EIME) in atopic dermatitis and psoriasis. Nat Immunol. 2018; 19(12):12861298.

30. Young CN, Koepke JI, Terlecky LJ, Borkin MS, Boyd SL, Terlecky SR. Reactive oxygen species in tumor necrosis factor-alpha-activated primary human keratinocytes: implications for psoriasis and inflammatory skin disease. J Invest Dermatol. 2008; 128(11):2606-2614.

31. Kumari S, Bonnet MC, Ulvmar MH, Wolk K, Karagianni N, Witte E, Uthoff-Hachenberg C, Renauld J$\mathrm{C}$, Kollias G, Toftgard R et al. Tumor necrosis factor receptor signaling in keratinocytes triggers interleukin24-dependent psoriasis-like skin inflammation in mice. Immunity. 2013; 39(5):899-911.

32. Xu F, Xu J, Xiong $X$, Deng Y. Salidroside inhibits MAPK, NF-KB, and STAT3 pathways in psoriasisassociated oxidative stress via SIRT1 activation. Redox Rep. 2019; 24(1):70-74.

33. Sano S, Chan KS, Carbajal S, Clifford J, Peavey M, Kiguchi K, Itami S, Nickoloff BJ, DiGiovanni J. Stat3 links activated keratinocytes and immunocytes required for development of psoriasis in a novel transgenic mouse model. Nat Med. 2005; 11(1):43-49.

34. Muller A, Dickmanns A, Resch C, Schakel K, Hailfinger S, Dobbelstein M, Schulze-Osthoff K, Kramer D. The CDK4/6-EZH2 pathway is a potential therapeutic target for psoriasis. J Clin Invest. 2020; 130(11):5765-5781. 
35. Yang L, Jin L, Ke Y, Fan X, Zhang T, Zhang C, Bian H, Wang G. E3 Ligase Trim21 Ubiquitylates and Stabilizes Keratin 17 to Induce STAT3 Activation in Psoriasis. J Invest Dermatol. 2018; 138(12):25682577.

36. Shi X, Jin L, Dang E, Chang T, Feng Z, Liu Y, Wang G. IL-17A upregulates keratin 17 expression in keratinocytes through STAT1- and STAT3-dependent mechanisms. J Invest Dermatol. 2011; 131(12):2401-2408.

37. Ilves M, Palomäki J, Vippola M, Lehto M, Savolainen K, Savinko T, Alenius H. Topically applied $\mathrm{ZnO}$ nanoparticles suppress allergen induced skin inflammation but induce vigorous IgE production in the atopic dermatitis mouse model. Part Fibre Toxicol. 2014; 11:38.

38. Nagajyothi PC, Cha SJ, Yang IJ, Sreekanth TVM, Kim KJ, Shin HM. Antioxidant and antiinflammatory activities of zinc oxide nanoparticles synthesized using Polygala tenuifolia root extract. J Photochem Photobiol B Biol. 2015; 146:10-17.

39. Chiricozzi A, Guttman-Yassky E, Suárez-Fariñas M, Nograles KE, Tian S, Cardinale I, Chimenti S, Krueger JG. Integrative responses to IL-17 and TNF-a in human keratinocytes account for key inflammatory pathogenic circuits in psoriasis. J Invest Dermatol. 2011; 131(3):677-687.

40. Yan Y, Liang H, Liu X, Liu L, Chen Y. Topical cationic hairy particles targeting cell free DNA in dermis enhance treatment of psoriasis. Biomaterials. 2021; 276:121027.

41. Svenskaya YI, Genina EA, Parakhonskiy BV, Lengert EV, Talnikova EE, Terentyuk GS, Utz SR, Gorin DA, Tuchin VV, Sukhorukov GB. A Simple Non-Invasive Approach toward Efficient Transdermal Drug Delivery Based on Biodegradable Particulate System. ACS Appl Mater Interfaces. 2019; 11(19):1727017282.

42. Kim JS, Song KS, Sung JH, Ryu HR, Choi BG, Cho HS, Lee JK, Yu IJ. Genotoxicity, acute oral and dermal toxicity, eye and dermal irritation and corrosion and skin sensitisation evaluation of silver nanoparticles. Nanotoxicology. 2013; 7(5):953-960.

43. Xu L, Wang Y-Y, Huang J, Chen C-Y, Wang Z-X, Xie H. Silver nanoparticles: Synthesis, medical applications and biosafety. Theranostics. 2020; 10(20):8996-9031.

44. Swindell WR, Michaels KA, Sutter AJ, Diaconu D, Fritz Y, Xing X, Sarkar MK, Liang Y, Tsoi A, Gudjonsson JE et al. Imiquimod has strain-dependent effects in mice and does not uniquely model human psoriasis. Genome Med. 2017; 9(1):24.

45. Chen B, Zhang C, Wang W, Chu Z, Zha Z, He X, Zhou W, Liu T, Wang H, Qian H. Ultrastable AgBiS2 Hollow Nanospheres with Cancer Cell-Specific Cytotoxicity for Multimodal Tumor Therapy. ACS Nano. 2020; 14(11):14919-14928. 
46. Chu T, Wu NL, Hsiao CY, Li HJ, Lin TY, Ku CH, Hung CF. An isoflavone extract from soybean cake suppresses 2,4-dinitrochlorobenzene-induced contact dermatitis. J Ethnopharmacol. 2020; 263:113037.

\section{Schemes}

Scheme 1 is in the supplementary files section.

\section{Figures}



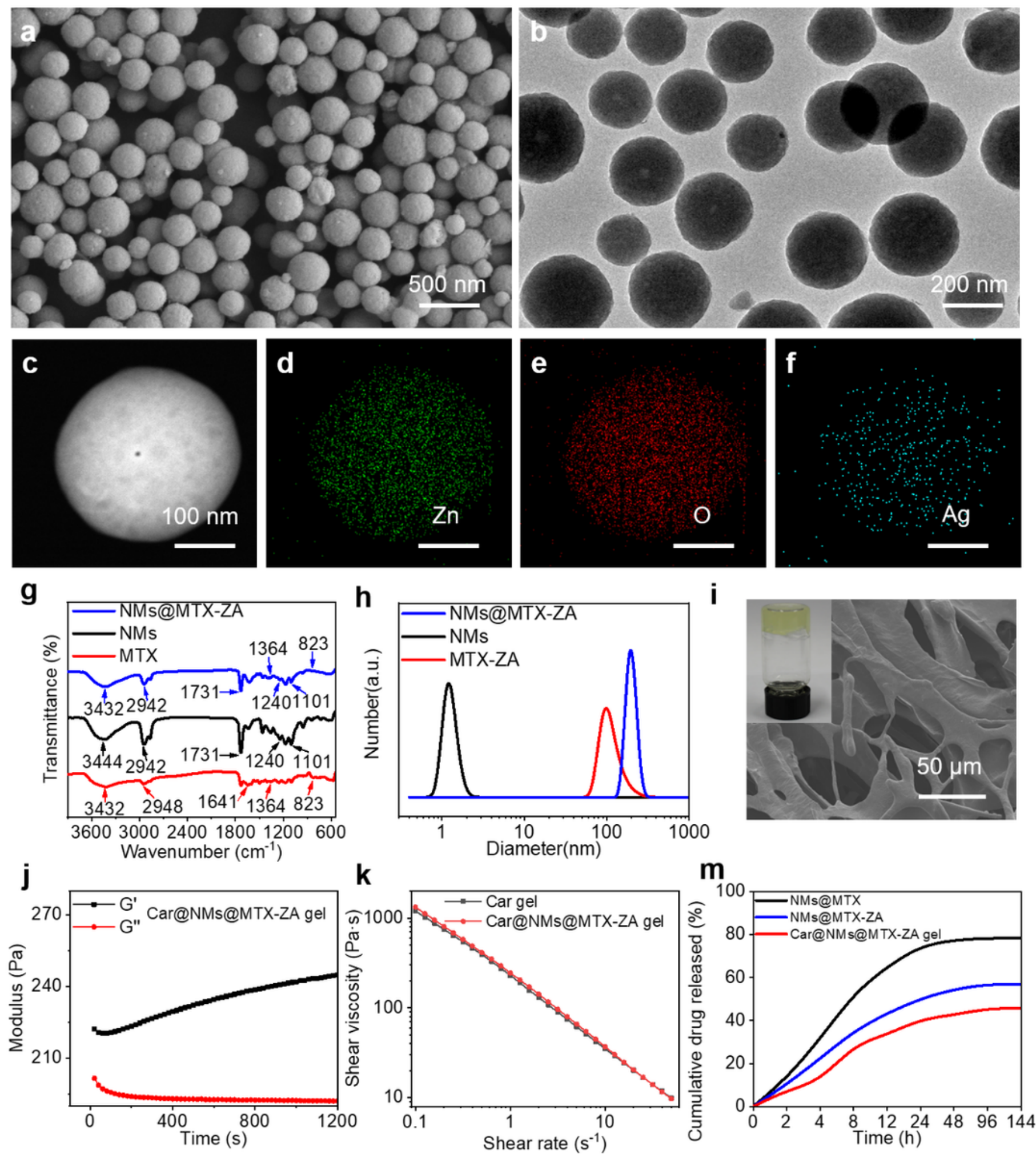

\section{Figure 1}

Characterization of the Car@NMs@MTX-ZA hydrogel. (a-b) SEM and TEM images of the ZnO/Ag hybrid microspheres. (c-f) STEM and elemental mapping images of the ZnO/Ag hybrid microspheres. (g) FT-IR spectra of NMs, MTX and NMs@MTX-ZA. (h) The size distributions of the NMs, MTX and NMs@MTX-ZA. (i) SEM image of porous structures and digital picture of the Car@NMs@MTX-ZA hydrogel. (j-k) 
Rheological properties of Car@NMs@MTX-ZA gel. (m) In vitro release profiles of MTX from different formulations at $37^{\circ} \mathrm{C}$ using phosphate buffered saline $(\mathrm{pH}=7.4)$. NMs: PCL-PEG-PCL.
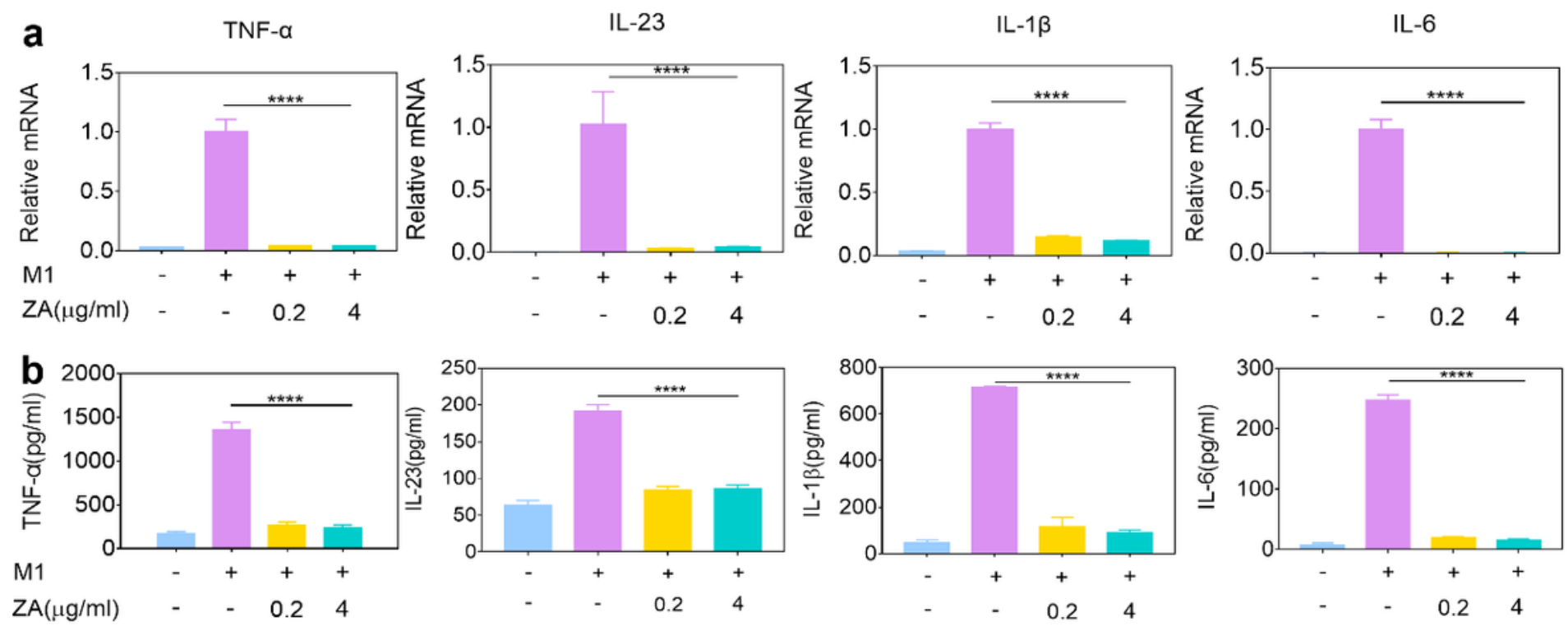

$\mathrm{ZA}(\mu \mathrm{g} / \mathrm{ml}) \quad-\quad-0.24$

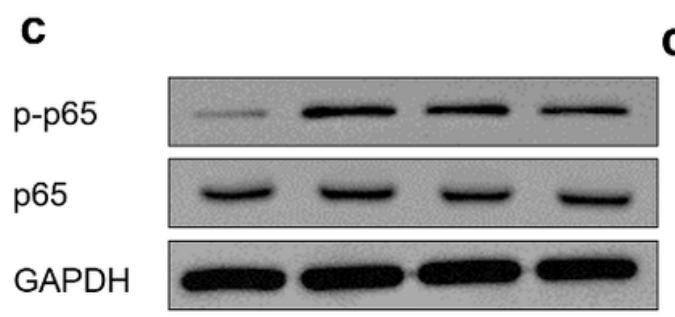

M1

$\mathrm{ZA}(\mu \mathrm{g} / \mathrm{ml})$
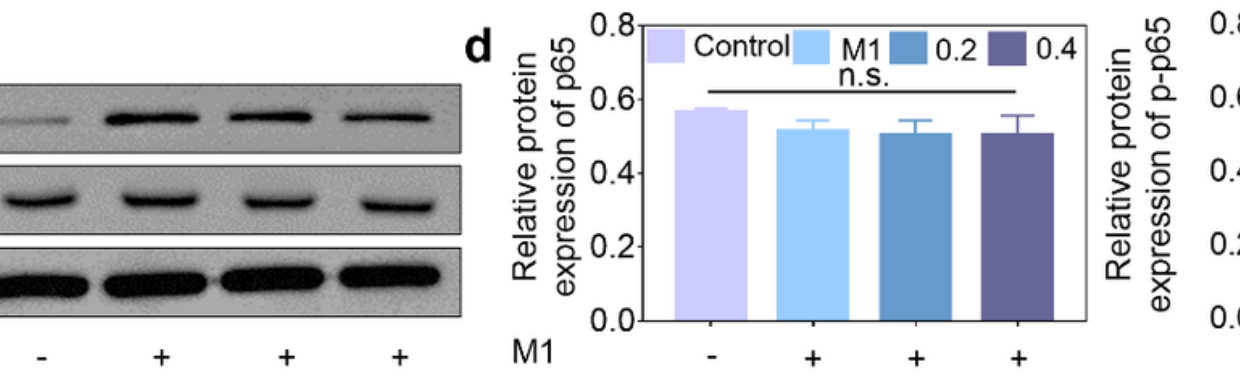

e

h-BD2

$\mathrm{ZA}(\mu \mathrm{g} / \mathrm{ml})$

0.24

S100A7

CXCL1
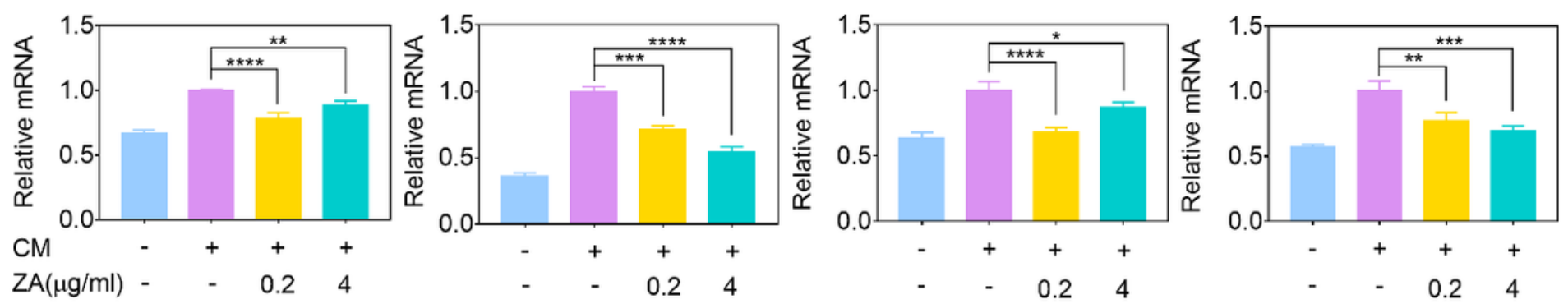

Figure 2

ZA attenuated innate cytokine profiles by reducing the phosphorylated form of p65. (a) mRNA and (b) protein levels of innate cytokine profiles from M1 cells measured by quantitative PCR and ELISA. (c-d) Quantitative analysis of the activation of phospho-p65 in M1 cells by western blotting. (e) Innate cytokine profiles expression in HaCaT cells with or without CM co-culture, analyzed by quantitative PCR. $n=3$, mean \pm SEM, n.s. (not significant), ${ }^{\star} p<0.05,{ }^{* \star} p<0.01,{ }^{* \star \star} p<0.001,{ }^{\star \star \star *} p<0.0001$ vs. M1-treated group, M1: LPS and IFN- $\gamma$, ZA: $0.1 \mathrm{wt} \% \mathrm{ZnO} / \mathrm{Ag}, \mathrm{CM}$ : conditional medium. 


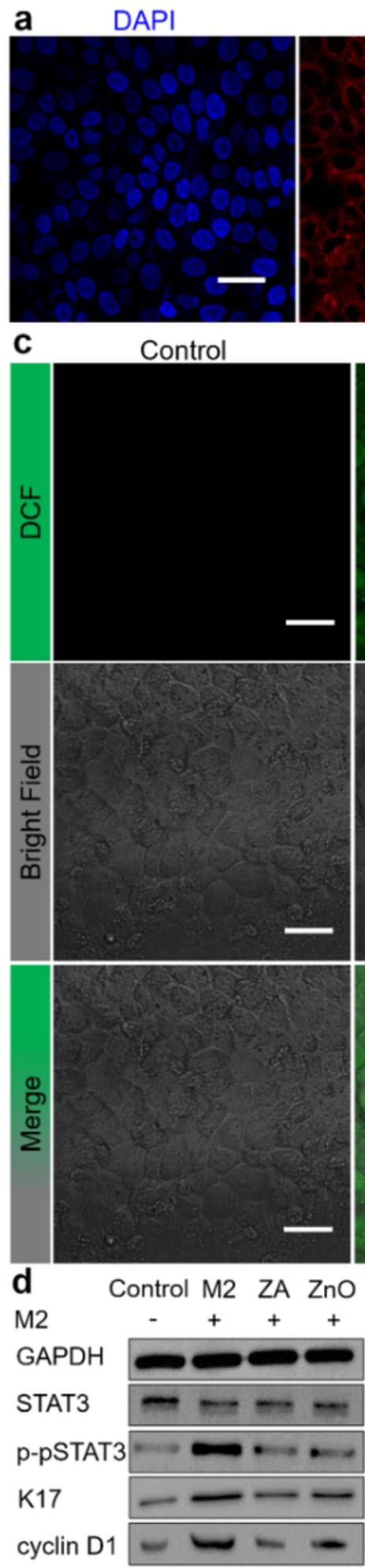

ZA

Merge

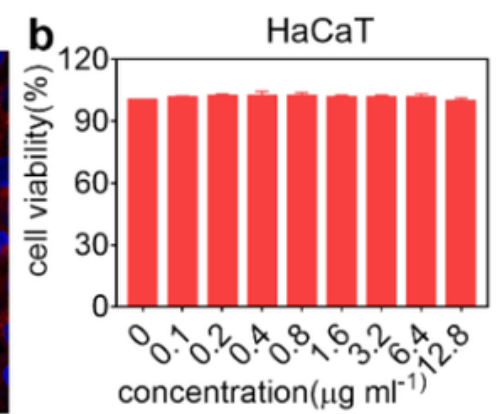

M2

ZA

$\mathrm{ZnO}$

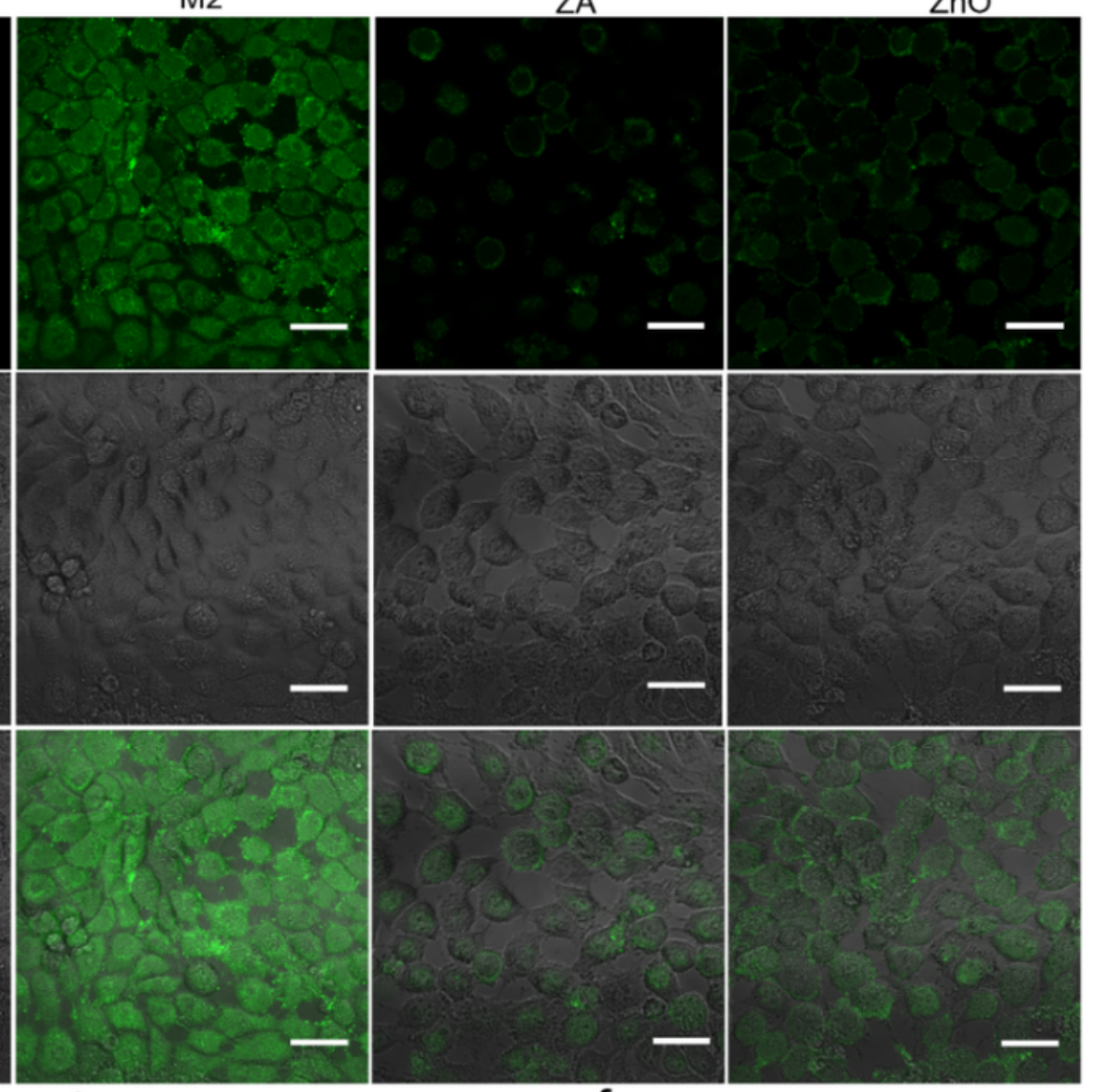

e Control $\mathrm{M} 2 \square \mathrm{ZA}=\mathrm{ZnO}$
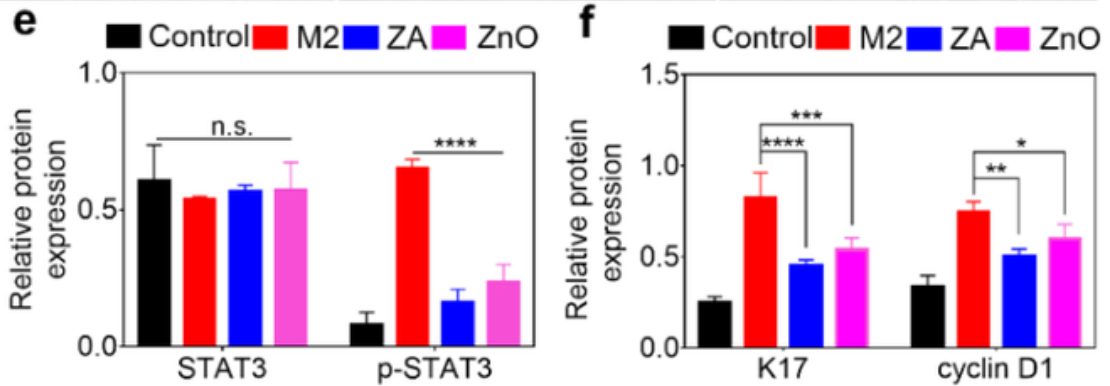

\section{Figure 3}

ZA inherent ablation of excessive ROS in KCs caused resistance to cyclin D1 expression and blocked selfamplifying K17/cytokine autoimmune loop. (a) CLSM images of HaCaT cells cellular internalization of NR-labeled ZA. Scale bar: $50 \mu \mathrm{m}$. (b) HaCaT cells viability after incubation with various concentrations of 
ZA determined by CCK-8 assays ( $n=8$, mean \pm SEM). (c) CLSM images of intracellular ROS levels (green) induced in HaCaT cells by M2 with DCFH-DA probes. Scale bar: $50 \mu \mathrm{m}$. (d-f) Quantitative analysis of the activation of phospho-STAT3 and its downstream genes in M2-treated HaCaT cells by western blotting (n $=3$, mean \pm SEM). n.s. (not significant), ${ }^{\star \star \star} p<0.001,{ }^{\star \star \star \star} p<0.0001 v s$. M2-treated group, M2: TNF-a and IL17A, ZA: $0.1 \mathrm{wt} \% \mathrm{ZnO} / \mathrm{Ag}$.

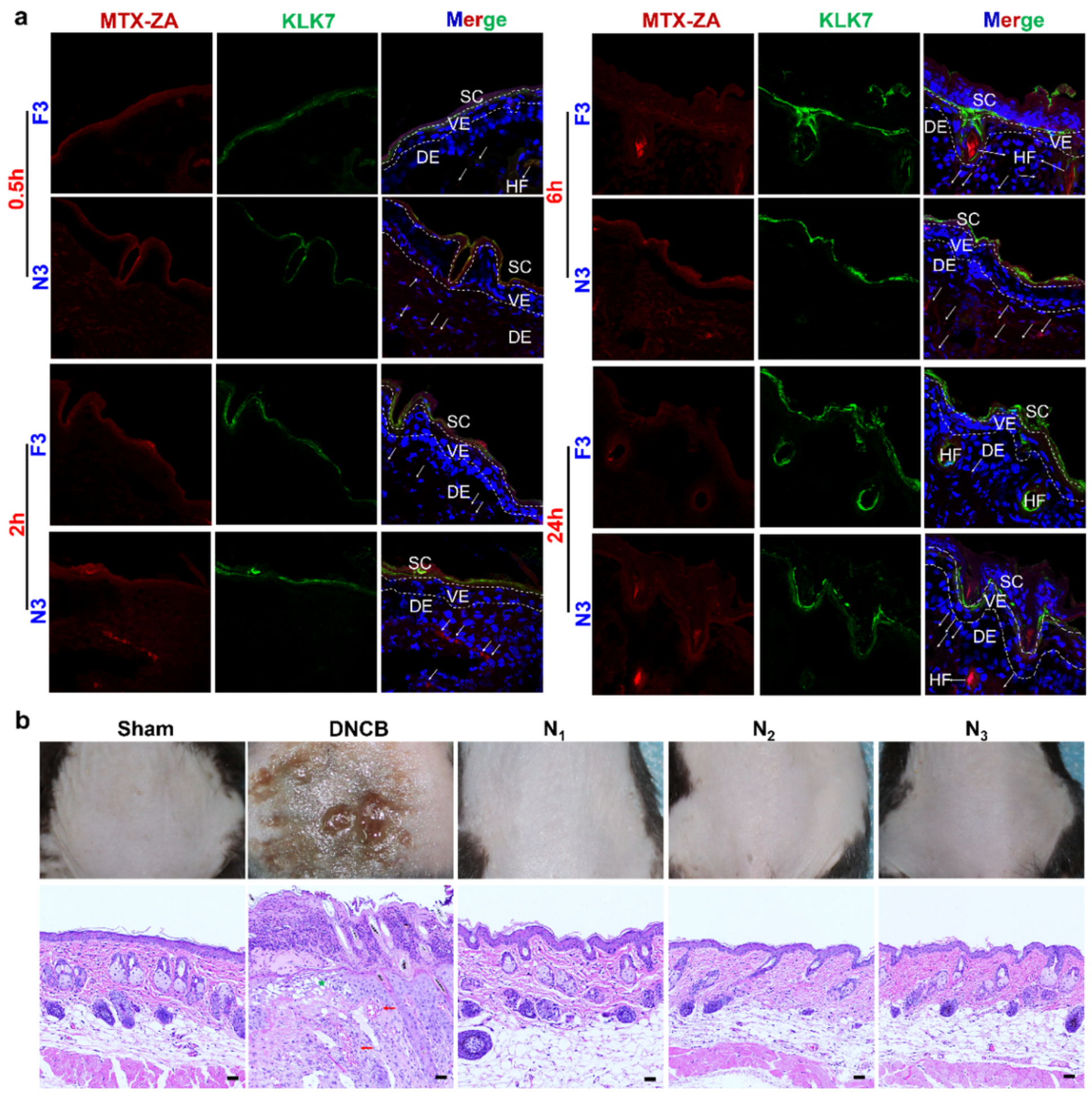


Skin permeability and irritation assessments of various formula hydrogels. (a) Penetration of NR-labeled MTX-ZA (red) through psoriatic skin at $0.5,2,6$, and $24 \mathrm{~h}$ after administration was observed by CLSM. (b) Photograph and histopathological images of mice back skin in skin irritation study (the green asterisk: confluent nests of apoptotic keratinocytes, the red arrows: perivascular infiltrate of lymphocytes). Scale bars:50 $\mu \mathrm{m}$, the nuclei were stained with DAPI (blue); KLK7 (green) was dyed to distinguish the SC layer, HF (hair follicles). The white arrows indicate NR-labeled MTX-ZA. Sham: control group, DNCB: 1\% 2,4dinitrochlorobenzene (DNCB)-induced contact dermatitis groups, $\mathrm{F}_{3}$ : Car@MTX-ZA hydrogel, $\mathrm{N}_{1}$ : Car@NMs@MTX hydrogel, N2: Car@NMs@ZA hydrogel, N3: Car@NMs@MTX-ZA hydrogel. 

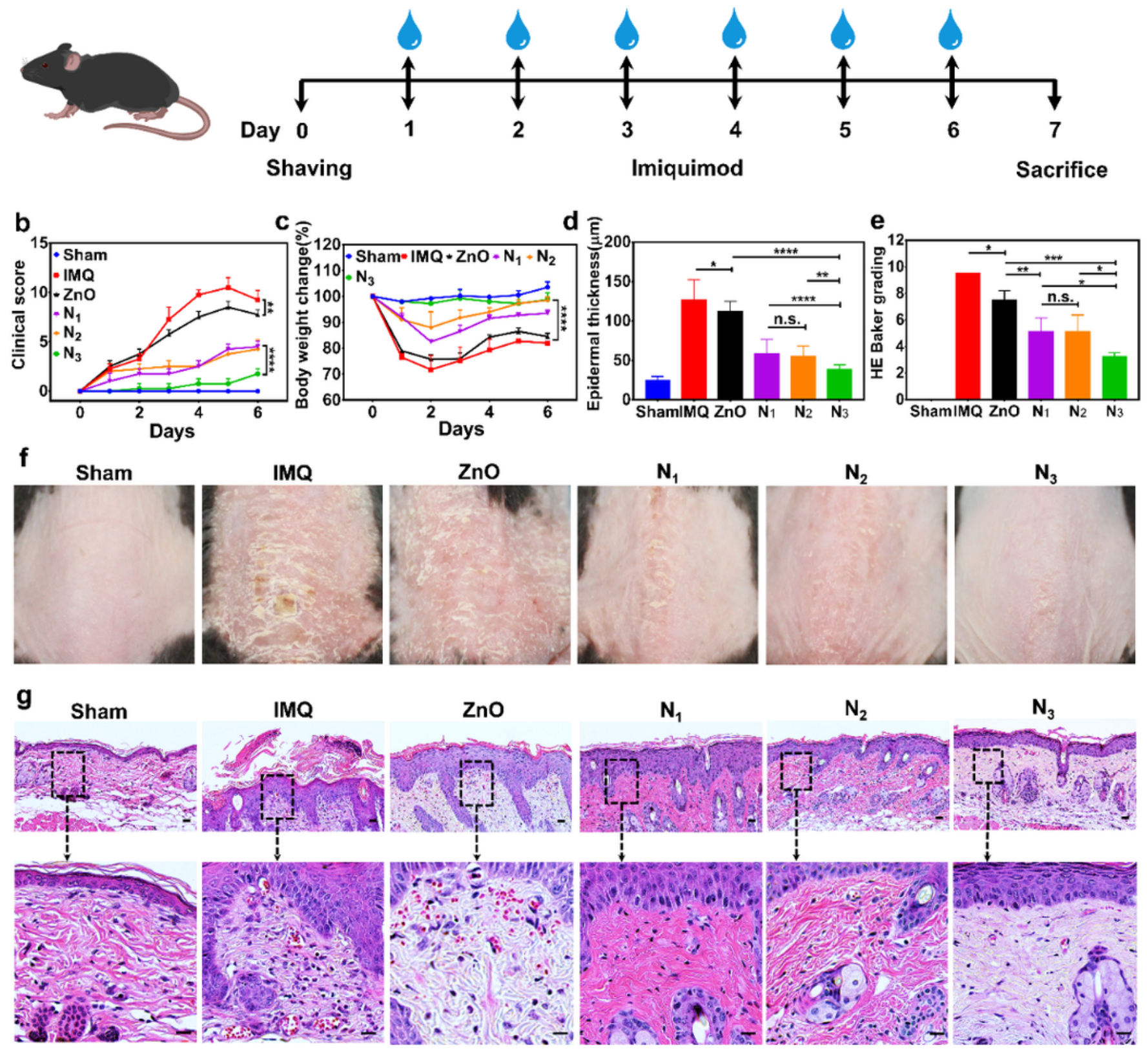

h

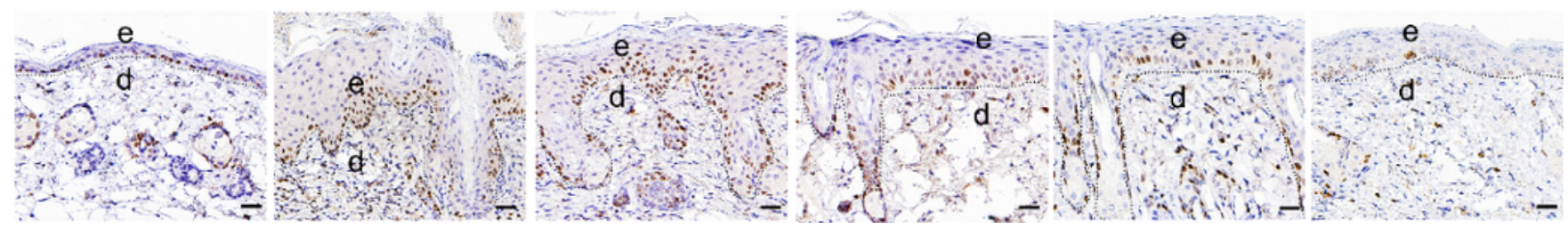

\section{Figure 5}

Transdermal therapy efficacy of various formula hydrogels on IMQ-induced psoriasiform skin inflammation. (a) Experimental scheme of antipsoriatic treatment. (b)PASI scores of skin lesions were monitored. (c) Change of body weight in percentage. (d) Quantification of the epidermal thickness. (e) HE Baker grading of the dorsal skin sections stained with H\&E. (f) Representative images of the dorsal skin. (g) Representative H\&E images of the dorsal skin sections. (h) Representative immunohistochemistry 
images of $\mathrm{KI} 7^{+}$cells in the dorsal skin sections. $\mathrm{n}=4$, mean $\pm \mathrm{SEM}$, scale bars: $20 \mu \mathrm{m}$, n.s. (not significant), ${ }^{*} p<0.05,{ }^{* \star} p<0.001,{ }^{* * \star *} p<0.0001$ vs. IMQ group, Sham: control group, IMQ: imiquimod group, $\mathrm{N}_{1}$ : Car@NMs@MTX hydrogel, N2: Car@NMs@ZA hydrogel, N3: Car@NMs@MTX-ZA hydrogel.

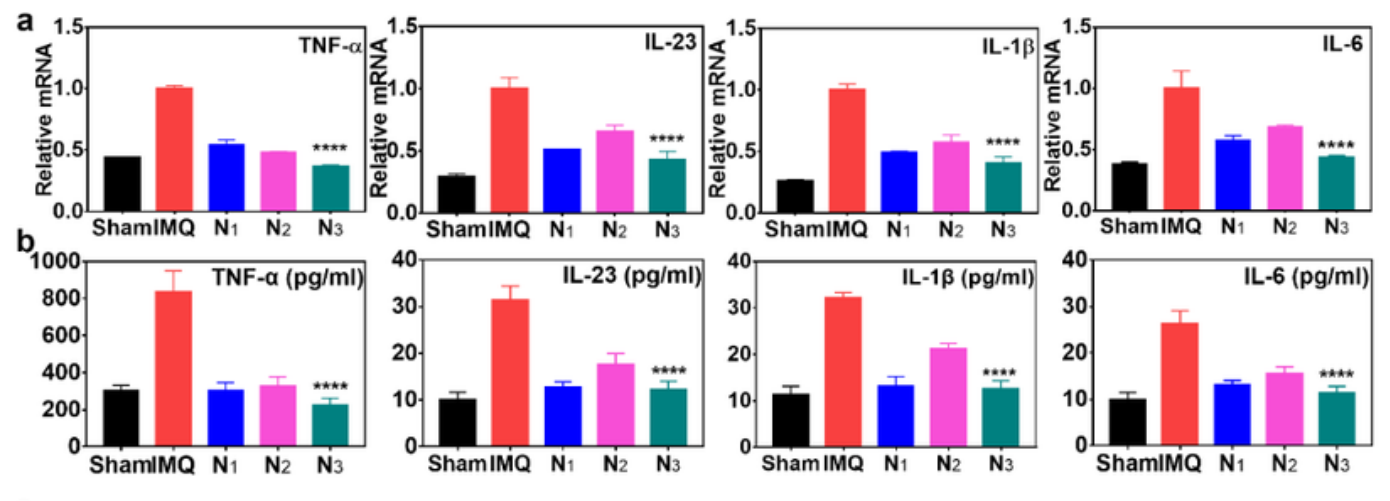

C

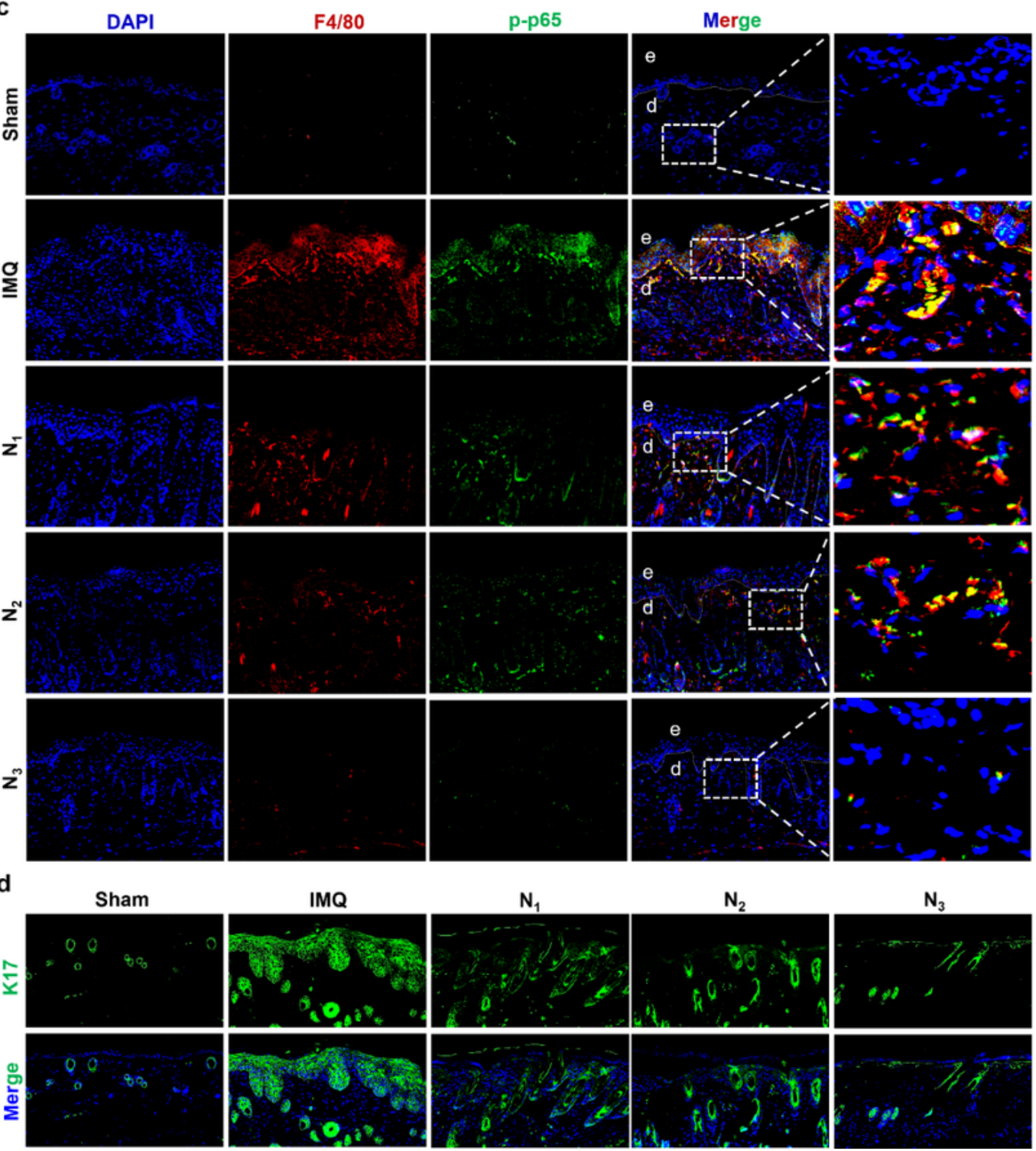

Figure 6 
Impediment of IMQ-induced inflammatory circuits caused by Car@NMs@MTX-ZA hydrogel. (a-b) qRTPCR and ELISA analysis of innate cytokine profiles in dorsal skin lesions after different treatments $(n=4$, mean \pm SEM). (c) Immunofluorescence co-staining of F4/80 (green) and p-p65 (red) in the dorsal skin lesions, scale bars:20 $\mu \mathrm{m}$. (d) Skin lesions were stained with K17 (green). Scale bars:20 $\mu \mathrm{m},{ }^{*} p<0.05$, ${ }^{* \star \star} p<0.001,{ }^{* \star \star \star} p<0.0001$ vs. IMQ group, sham: control group, IMQ: imiquimod group, $\mathrm{N}_{1}$ : Car@NMs@MTX hydrogel, $\mathrm{N}_{2}$ : Car@NMs@ZA hydrogel, N3: Car@NMs@MTX-ZA hydrogel.

\section{Supplementary Files}

This is a list of supplementary files associated with this preprint. Click to download.

- GraphicalTableofContents.docx

- 20220114SupplementaryInformationXuJM.docx

- floatimage1.jpeg 